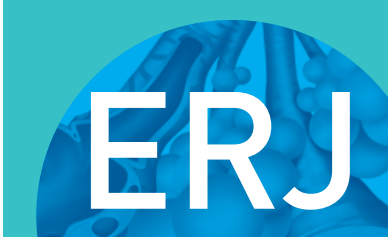

open research

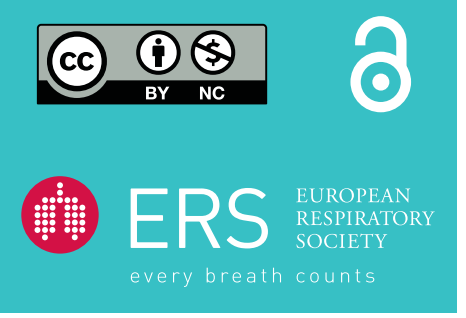

\section{Comparison of the QuantiFERON-TB and tuberculin skin test for detection of latent tuberculosis infection in cancer patients in a developing country}

\author{
Javier Leonardo Galindo (10)', Adriana Catalina Galeano (1)', \\ David Alfonso Suarez-Zamora (102, Ana Milena Callejas (101, \\ Mónica Patricia Caicedo-Verástegui ${ }^{3,4}$, Darío Londoño ${ }^{5,4}$, Luis Gerardo García- \\ Herreros $^{6,4}$, Aylen Vanessa Ospina-Serrano ${ }^{7,4}$, Alfredo Saavedra ${ }^{1,8}$, \\ Plutarco Garcíaherreros ${ }^{8}$, Diana María Palacios ${ }^{2,4}$ and Margarita Baldión (10 2,4
}

Affiliations: ${ }^{1}$ Dept of Internal Medicine, Universidad Nacional de Colombia, Bogotá DC, Colombia. ${ }^{2}$ Dept of Pathology and Laboratories, Fundación Santa Fe de Bogotá, Bogotá DC, Colombia. ${ }^{3}$ Dept of Respiratory and Critical Care Medicine, Fundación Santa Fe de Bogotá, Bogotá DC, Colombia. "School of Medicine, Universidad de los Andes, Bogotá DC, Colombia. ${ }^{5}$ Dept of Public Health, Fundación Santa Fe de Bogotá, Bogotá DC, Colombia. 'Dept of Thoracic Surgery, Fundación Santa Fe de Bogotá, Bogotá DC, Colombia. ${ }^{7}$ Carlos Ardila Lülle Oncology Institute, Fundación Santa Fe de Bogotá, Bogotá DC, Colombia. ${ }^{8}$ Dept of Thoracic Clinic, Instituto Nacional de Cancerología, Bogotá DC, Colombia.

Correspondence: Ana M. Baldión Elorza, Dept of Pathology and Laboratories, Fundación Santa Fe de Bogotá, Carrera 7 \# 117-15 (Second Floor), Bogotá DC, Colombia. E-mail: ana.baldionafsfb.org.co

ABSTRACT Cancer patients have an increased risk of reactivation of latent tuberculosis infection. It is unknown which strategy on screening should be used in this population in developing countries. We aimed to determine the concordance between the tuberculin skin test (TST) and QuantiFERON ${ }^{\circ}$ TB (QFT) assay in order to diagnose latent tuberculosis infection in cancer patients.

We conducted a cross-sectional study of the agreement of diagnostic tests. Prevalence and agreement between tests were calculated. A logistic regression to assess predictors of discordance was performed. The accuracy of the TST to predict QFT results by a receiver operating characteristic (ROC) curve was evaluated.

We included 149 adults with cancer without active tuberculosis. Prevalence of latent tuberculosis infection was $21.5 \%(n=32)$, defined as positive results on either test. Test agreement was moderate for the diagnosis of latent tuberculosis infection $(\kappa=0.43,90 \%$ CI $0.26-0.6)$. No predictor was associated with the chance of discordant results. Agreement improved slightly using a cut-off point $\geqslant 8 \mathrm{~mm}(\kappa=0.5,90 \%$ CI $0.35-0.66)$.

In a moderate-incidence setting, a moderate agreement was found between tests in cancer patients. Modification of the cut-off points of test results achieved marginally better agreement between the TST and QFT.

@ERSpublications

QuantiFERON and tuberculin skin test have a moderate agreement for latent tuberculosis infection diagnosis in cancer patients. Modifying cut-off points may improve agreement of tests. http://bit.ly/32bNwLc

Cite this article as: Galindo JL, Galeano AC, Suarez-Zamora DA, et al. Comparison of the QuantiFERON-TB and tuberculin skin test for detection of latent tuberculosis infection in cancer patients in a developing country. ERJ Open Res 2019; 5: 00258-2018 [https://doi.org/10.1183/ 23120541.00258-2018].

The dataset generated and analysed during the current study are available in the Figshare repository: https://oi.org/10. 6084/m9.figshare.5821314.

Received: Dec 272018 | Accepted after revision: May 282019

Copyright $\odot E R S$ 2019. This article is open access and distributed under the terms of the Creative Commons Attribution Non-Commercial Licence 4.0. 


\section{Introduction}

Tuberculosis remains a leading cause of mortality related to infectious diseases worldwide. An estimated of 10 million people developed tuberculosis in 2017, resulting in 1.3 million deaths [1]. Active tuberculosis will develop in $7.7 \%$ of people with a latent tuberculosis infection (LTBI) after the first year following infection and $14.2 \%$ by the end of year 20 [2]. Previous studies have found that risk factors such as HIV infection, malnutrition, use of immunosuppressive drugs, and cancer, especially haematologic, head and neck or pulmonary malignancies, contribute to significantly increase the tuberculosis reactivation rate [3-5].

The diagnosis of LTBI can be challenging, especially in some high-risk populations. Tests available for LTBI diagnosis are the tuberculin skin test (TST) and the interferon- $\gamma$ release assays (IGRAs) [6]. TST is based on a delayed hypersensitivity response to Mycobacterium tuberculosis antigens, assessing host sensitisation to a prior exposure to mycobacteria [7]. Cut-off points for interpretation of the results have been set according to the risk of reactivation of each population [7, 8]. M. tuberculosis shares several antigens with Mycobacterium bovis and other nontuberculous mycobacteria; prior vaccination with Bacillus Calmette-Guérin (BCG) increases the risk of false positives of the TST [9, 10]. Two IGRAs are available, QuantiFERON ${ }^{\circ}$-TB (QFT) and T-SPOT ${ }^{\circ}$.TB [11]. QFT Gold In-Tube, the third generation of the assay, is an ELISA-based measurement of the interferon- $\gamma$ released by lymphocytes on exposure to ESAT-6, CFP-10, and TB7.7 antigens [9, 11]. IGRAs offer some advantages over the TST because they require a single visit for testing and the antigens used are not expressed by M. bovis [9, 11]. However, IGRAs may present false-positive results by cross-reactivity with some nontuberculous mycobacteria or manufacturing issues, and a higher cost [11]. In addition, both TST and IGRAs may have false-negative results in immunosuppressed individuals $[7,9]$.

Neither TST nor IGRAs can distinguish between the cure or persistence of bacteria in a state of latency; they only reflect the host's immune memory against bacteria [6]. Due to the lack of a gold standard for the diagnosis of LTBI, the performance of these tests has been estimated using different approaches such as assessing their agreement [11]. A poor agreement between tests would affect the estimation of the prevalence of LTBI and would suggest that these tests cannot be used interchangeably for diagnosis. The aim of the present study was to evaluate the agreement between the TST and QFT for the diagnosis of LTBI in individuals with malignancies from a developing country.

\section{Methods}

Study design and data collection

This was a study of the agreement of diagnostic tests conducted in a consecutive sample of patients with cancer at two tertiary care hospitals in Colombia. The TST was carried out using the Mantoux technique; a positive result was defined as an induration $\geqslant 10 \mathrm{~mm}$ measured after $72 \mathrm{~h}$, according to the recommendations for oncological patients [8]. QFT Gold In-Tube (Cellestis Limited, Carnegie, Australia) was measured fulfilling the manufacturer's quality recommendations.

The staff responsible for carrying out each of the tests measured them independently; they were blinded to the data provided by the patients. The QFT was measured first, followed by TST to avoid false positives of the IGRA due to a possible boosting effect of tuberculin. Both tests were performed the same day to avoid changes in the clinical cancer status. Results were categorised as discordant if there was a TST positive with a QFT negative or a TST negative with a QFT positive.

Enrolled patients completed a detailed questionnaire about clinical information and risk factors for exposure to tuberculosis. Evidence of a BCG scar was used as a proof of BCG vaccination.

\section{Inclusion criteria}

Patients 18 years or older with a diagnosis of cancer were included, regardless of clinical status or type. We excluded patients who had cough in the last 2 weeks, chest images in the last 3 months suggestive of pulmonary tuberculosis, or fever or involuntary weight loss that did not have studies to rule out active tuberculosis. Patients with a prior history of tuberculosis, HIV infection, hereditary immunodeficiencies, renal replacement therapy, pregnancy, or BCG intravesical immunotherapy for bladder cancer were excluded.

\section{Sample size}

The sample size was computed knowing the sensitivity of these tests and the agreement among them from the published studies of LTBI in cancer patients [12-14]. An expected Cohen $\kappa$ statistic of 0.5 was set to identify at least a moderate agreement between tests. As a result, it was estimated that it would be necessary to enrol 168 participants in order to achieve an absolute precision of 0.12 and a $90 \%$ confidence level. The sample size was computed using Epidat version 4.1 (Dirección Xeral de Saúde Pública da Xunta de Galicia, Spain) [15]. 


\section{Statistical analysis}

A descriptive analysis of the variables of interest was conducted to report the categorical data by the distribution of frequencies, relative frequencies, and proportions; for the continuous variables, the results were reported by median and the interquartile range or by mean and the standard deviation, depending on the distribution.

Indeterminate QFT results were omitted from the agreement analysis. The strength of agreement was set according to the Landis and Koch classification as "slight" if $\kappa$ was $\leqslant 0.2$, as "fair" between 0.21 and 0.4 , as "moderate" between 0.41 and 0.6 , as "substantial" between 0.61 and 0.8 , and as "almost perfect" $\geqslant 0.81$ [16] Factors statistically associated with the chance of obtaining a discordant result between the tests ( $\mathrm{p}$-values on univariate analysis $<0.2$ ) were included in the multiple analysis by the logistic regression method.

The cut-off points of the TST were explored to predict the QFT results using its area under the receiver operating characteristic (ROC) curve. The antigenic response of the QFT in correspondence to the TST results was described; for comparison of the TST and QFT, antigen responses minus the nil value $>10 \mathrm{IU} \cdot \mathrm{mL}^{-1}$ were truncated at $10 \mathrm{IU} \cdot \mathrm{mL}^{-1}$. A sensitivity analysis was performed to calculate the test agreement after changing the TST cut-off point according to the ROC curve data and the QFT cut-off point according to the antigenic response values.

All reported p-values were two-tailed and calculated with statistical significance set to $\mathrm{p}<0.05$. Statistical analyses were performed using Stata version 12.0 (StataCorp LP, TX, USA).

\section{Ethics}

All patients were provided with a written informed consent form to authorise data collection and testing. The study was approved by the local ethics committees of Fundación Santa Fe de Bogotá and Instituto Nacional de Cancerología, in accordance with the principles of the Declaration of the Helsinki, World Medical Association and the Guideline for Good Clinical Practice. The information provided by the patients was confidential, but due to the potential preventive benefit of test results, their doctors were notified if the results were positive.

\section{Results \\ Study population}

From March 2015 to January 2017, 149 participants underwent testing. Enrolment was suspended because the laboratory changed the QFT assay. This sample size achieved a $\kappa$ precision of 0.127 . The sample was comprised of individuals with a mean of 62 years (range: 18 to 91 years). A total of 100 (67.1\%) patients had a scar from the BCG vaccine. The most common types of malignancies were soft tissues and breast (20.1\%), followed by thyroid (18.8\%), gastrointestinal (14.8\%) and haematologic (10.1\%). Most individuals had a cancer status of partial or complete remission (41.6\%); more than one-third were receiving palliative chemotherapy $(22.8 \%)$, curative chemotherapy $(4.7 \%)$ or other kinds of treatments $(10.7 \%)$, which mainly consisted of levothyroxine therapy for thyroid cancers.

Factors associated with immunosuppression were found in 54 (36.2\%) patients, such as the use of immunosuppressive therapies (mainly selective inhibitors of tyrosine kinase receptors and hormone therapy), chronic use of corticosteroids and haematopoietic stem cell transplantation (table 1).

\section{Prevalence and test agreement}

The TST had $22(14.8 \%)$ positive results, QFT had 21 (14.1\%) positive results, and only 11 patients $(7.4 \%)$ were positive by both tests. A total of 14 patients presented with skin reactions to tuberculin without meeting the threshold of positivity $(\geqslant 10 \mathrm{~mm})$, and 4 of them presented a positive QFT result. Prevalence of LTBI was $21.5 \%$ (95\% CI 15.6-28.9\%), defined as a positive result on either test independently of its agreement.

We found 21 discordant results, distributed across 11 cases of TST positive/QFT negative and 10 cases of QFT positive/TST negative. Overall, nine TST positive/QFT negative patients and five QFT positive/TST negative patients were BCG vaccinated. The overall agreement between tests was $86 \%$, a chance-adjusted agreement was moderate ( $\kappa=0.43,90 \%$ CI $0.26-0.6)$ (table 2 ).

There was no difference in the distribution of TST or QFT positive results by type of cancer or by its status in the logistic regression analysis. Although the BCG vaccination was common, it did not yield a difference in the frequency of the test results. No risk factors were found associated with the chance of a discordant result between tests in the univariate analysis (table 3 ). 
TABLE 1 Characteristics of the study population stratified by the tuberculin skin test (TST) and the QuantiFERON-TB results

\begin{tabular}{|c|c|c|c|c|c|}
\hline & Total & \multicolumn{2}{|c|}{ TST } & \multicolumn{2}{|c|}{ QuantiFERON-TB } \\
\hline Patients & 149 & 22 & 127 & 21 & 127 \\
\hline Age years median (interquartile range) & $62(54-70)$ & $59(53-66)$ & $62(54-70)$ & $64(63-73)$ & $60(53-69)^{\#}$ \\
\hline Female & $94(63.1 \%)$ & $12(54.5 \%)$ & $82(64.6 \%)$ & $11(52.4 \%)$ & $82(64.6 \%)$ \\
\hline \multicolumn{6}{|l|}{ Type of cancer diagnosis by location } \\
\hline Soft tissues and breast & $30(20.1 \%)$ & $4(18.2 \%)$ & $26(20.5 \%)$ & $3(14.3 \%)$ & $27(21.3 \%)$ \\
\hline Haematologic & $15(10.1 \%)$ & $3(13.6 \%)$ & $12(9.4 \%)$ & $3(14.3 \%)$ & $12(9.4 \%)$ \\
\hline Renal and urinary tract & $13(8.7 \%)$ & $2(9.1 \%)$ & $11(8.7 \%)$ & $3(14.3 \%)$ & $10(7.9 \%)$ \\
\hline Lung & $9(6 \%)$ & $2(9.1 \%)$ & $7(5.5 \%)$ & $1(4.8 \%)$ & $8(6.3 \%)$ \\
\hline Gynaecological & $9(6 \%)$ & $2(9.1 \%)$ & $7(5.5 \%)$ & $1(4.8 \%)$ & $8(6.3 \%)$ \\
\hline Head and neck (not thyroid) & $6(4 \%)$ & $1(4.5 \%)$ & $5(3.9 \%)$ & $1(4.8 \%)$ & $5(3.9 \%)$ \\
\hline Other & $17(11.4 \%)$ & $2(9.1 \%)$ & $15(11.8 \%)$ & $1(4.8 \%)$ & $16(12.6 \%)$ \\
\hline Progression, with follow-up & $18(12.1 \%)$ & $4(18.2 \%)$ & $14(11 \%)$ & $2(9.5 \%)$ & $16(12.6 \%)$ \\
\hline Initial staging, before the beginning of treatment & $10(6.7 \%)$ & $2(9.1 \%)$ & $8(6.3 \%)$ & $2(9.5 \%)$ & $8(6.3 \%)$ \\
\hline Current management with curative chemotherapy & $7(4.7 \%)$ & & $7(5.5 \%)$ & & $6(4.7 \%)$ \\
\hline Missing results & $2(1.3 \%)$ & & & & \\
\hline Scar from BCG vaccine & $100(67.1 \%)$ & $14(63.6 \%)$ & $86(67.7 \%)$ & $11(52.4 \%)$ & $89(70.1 \%)$ \\
\hline \multicolumn{6}{|l|}{ Comorbidities associated with immunosuppression } \\
\hline Treatment with other immunosuppressants in the last 3 months & $35(23.5 \%)$ & $4(18.2 \%)$ & $31(24.4 \%)$ & $3(14.3 \%)$ & $31(24.4 \%)$ \\
\hline Corticosteroid use for $>90$ days, $>15 \mathrm{mg}$ prednisone & $4(2.7 \%)$ & & $4(3.1 \%)$ & & $4(3.1 \%)$ \\
\hline Insulin-dependent diabetes & $3(2 \%)$ & & $3(2.4 \%)$ & & $3(2.4 \%)$ \\
\hline History of haematopoietic stem cell transplant & $2(1.3 \%)$ & $1(4.5 \%)$ & $1(0.8 \%)$ & & $2(1.6 \%)$ \\
\hline Chronic kidney disease & $2(1.3 \%)$ & & $2(1.6 \%)$ & & $2(1.6 \%)$ \\
\hline Cases with two comorbidities & $8(5.4 \%)$ & $2(9.1 \%)$ & $6(4.7 \%)$ & $3(14.3 \%)$ & $5(3.9 \%)$ \\
\hline
\end{tabular}

The indeterminate results of QuantiFERON-TB were excluded for statistical analyses. BCG: bacille Calmette-Guérin. ${ }^{\#}$ : $p=0.02$, logistic regression between QuantiFERON-TB negative and positive results groups; ๆ : lymphocyte count $<1000$ per $\mathrm{mm}^{3}$.

\section{Cut-off point variation of tests}

Comparison between the tests was explored using the QFT as a gold standard. The area under the ROC curve of the TST was 0.81 to predict QFT results (figure 1). The TST better predicted the QFT results using a cut-off point $\geqslant 8 \mathrm{~mm}$, with a sensitivity of $66.7 \%$ and a specificity of $89.8 \%$ (Table 4 ). The agreement of tests using the cut-off point $\geqslant 8 \mathrm{~mm}$ improved slightly ( $\kappa=0.5,90 \%$ CI $0.35-0.66$ ).

Regarding the measurement of interferon- $\gamma$ by QFT, the median of antigen response minus the nil value in individuals with positive TST was $1.06 \mathrm{IU} \cdot \mathrm{mL}^{-1}$, whereas in individuals with negative TST was $0 \mathrm{IU} \cdot \mathrm{mL}^{-1}$ $(\mathrm{p}<0.0001)$ (figure 2). Overall, $96.9 \%$ of the results in TST negative individuals were less than $1 \mathrm{IU} \cdot \mathrm{mL}^{-1}$, while $92.1 \%$ of the results were less than $0.35 \mathrm{IU} \cdot \mathrm{mL}^{-1}$. The agreement between the cut-off point $\geqslant 1 \mathrm{IU} \cdot \mathrm{mL}^{-1}$

TABLE 2 Distribution of the tuberculin skin test (TST) and QuantiFERON-TB results in the population

TST

\begin{tabular}{lc|}
\cline { 2 - 2 } & Negative \\
\hline Negative & $116(91.3 \%)$ \\
Positive & $11(50 \%)$ \\
Total & $127(85.2 \%)$ \\
\hline
\end{tabular}

QuantiFERON-TB

Positive Indeterminate

$10(7.9 \%)$
$11(50 \%)$
$21(14.1 \%)$

$1(0.8 \%)$

$1(0.7 \%)$
Total 


\begin{tabular}{|c|c|c|}
\hline & OR $(95 \% \mathrm{CI})$ & p-value \\
\hline \multicolumn{3}{|l|}{ Age } \\
\hline$<65$ years & 1 & \multirow[t]{2}{*}{0.92} \\
\hline$\geqslant 65$ years & $1.05(0.4-2.71)$ & \\
\hline \multicolumn{3}{|l|}{ Sex } \\
\hline Female & 1 & \multirow[t]{2}{*}{0.92} \\
\hline Male & $1.05(0.4-2.71)$ & \\
\hline \multicolumn{3}{|l|}{ Type of cancer diagnosis by location } \\
\hline Haematologic & $0.92(0.19-4.42)$ & \multirow[t]{2}{*}{0.92} \\
\hline Nonhaematologic $\#$ & 1 & \\
\hline \multicolumn{3}{|l|}{ Cancer status } \\
\hline Partial or complete remission & 1 & \multirow[t]{5}{*}{0.84} \\
\hline Current management with palliative or curative chemotherapy" & $1.67(0.54-5.18)$ & \\
\hline Current management with other treatments & $1.81(0.41-7.98)$ & \\
\hline Progression, with follow-up & $0.98(0.19-5.2)$ & \\
\hline Initial staging, before the beginning of treatment & $1.96(0.35-11.17)$ & \\
\hline \multicolumn{3}{|l|}{ Scar of BCG vaccine } \\
\hline Yes & $0.65(0.25-1.71)$ & \multirow[t]{2}{*}{0.39} \\
\hline No & 1 & \\
\hline \multicolumn{3}{|l|}{ Comorbidities associated with immunosuppression } \\
\hline Treatment with other immunosuppressants & $1.98(0.69-5.78)$ & \multirow[t]{5}{*}{0.43} \\
\hline Corticosteroid use for $>90$ days & $4.25(0.69-26.22)$ & \\
\hline Insulin-dependent diabetes & $2.83(0.27-29.9)$ & \\
\hline History of haematopoietic stem cell transplant & $2.83(0.27-29.9)$ & \\
\hline None & 1 & \\
\hline \multicolumn{3}{|l|}{ Lymphopenia } \\
\hline Yes & $0.99(0.26-3.81)$ & 0.99 \\
\hline No & 1 & \\
\hline
\end{tabular}

BCG: bacille Calmette-Guérin. \#: nonhaematologic cancers were grouped for analysis; ": cases of management with palliative and curative chemotherapy were grouped for analysis.

and the TST $(\geqslant 10 \mathrm{~mm})$ was slightly higher $(\kappa=0.54,90 \%$ CI $0.37-0.71)$, suggesting that a higher cut-off point than the threshold recommended $\left(\geqslant 0.35 \mathrm{IU} \cdot \mathrm{mL}^{-1}\right.$ and $\geqslant 25 \%$ of the nil value) could better discriminate the results. The cut-off point $\geqslant 0.35 \mathrm{IU} \cdot \mathrm{mL}^{-1}$ had a lower specificity to predict TST results (sensitivity $50 \%$ and specificity $92.1 \%$ ) than the cut-off point $\geqslant 1 \mathrm{IU} \cdot \mathrm{mL}^{-1}$ (sensitivity $50 \%$ and specificity $96.9 \%$ ).

TABLE 4 Sensitivity, specificity, and likelihood ratios (LRs) for different tuberculin skin test (TST) cut-off points correctly identifying the QuantiFERON-TB results

\begin{tabular}{|c|c|c|c|c|c|}
\hline TST mm & Sensitivity & Specificity & Correctly classified & $\mathrm{LR}^{+}$ & $\mathrm{LR}^{-}$ \\
\hline$\geqslant 0$ & $100 \%$ & $0 \%$ & $14.19 \%$ & 1 & \\
\hline$\geqslant 3$ & $71.43 \%$ & $83.46 \%$ & $81.76 \%$ & 4.32 & 0.34 \\
\hline$\geqslant 4$ & $71.43 \%$ & $85.04 \%$ & $83.11 \%$ & 4.77 & 0.34 \\
\hline$\geqslant 5$ & $66.67 \%$ & $85.83 \%$ & $83.11 \%$ & 4.7 & 0.39 \\
\hline$\geqslant 6$ & $66.67 \%$ & $87.4 \%$ & $84.46 \%$ & 5.29 & 0.38 \\
\hline$\geqslant 7$ & $66.67 \%$ & $88.98 \%$ & $85.81 \%$ & 6.05 & 0.37 \\
\hline$\geqslant 8$ & $66.67 \%$ & $89.76 \%$ & $86.49 \%$ & 6.51 & 0.37 \\
\hline$\geqslant 9$ & $57.14 \%$ & $90.55 \%$ & $85.81 \%$ & 6.05 & 0.47 \\
\hline$\geqslant 10$ & $52.38 \%$ & $91.34 \%$ & $85.81 \%$ & 6.05 & 0.52 \\
\hline$\geqslant 11$ & $47.62 \%$ & $93.70 \%$ & $87.16 \%$ & 7.56 & 0.56 \\
\hline$\geqslant 12$ & $47.62 \%$ & $96.85 \%$ & $89.86 \%$ & 15.12 & 0.54 \\
\hline$\geqslant 13$ & $42.86 \%$ & $97.64 \%$ & $89.86 \%$ & 18.14 & 0.59 \\
\hline$\geqslant 14$ & $38.1 \%$ & $99.21 \%$ & $90.54 \%$ & 48.38 & 0.62 \\
\hline$\geqslant 15$ & $33.33 \%$ & $100 \%$ & $90.54 \%$ & & 0.67 \\
\hline$\geqslant 16$ & $23.81 \%$ & $100 \%$ & $89.19 \%$ & & 0.76 \\
\hline$\geqslant 20$ & $19.05 \%$ & $100 \%$ & $88.51 \%$ & & 0.81 \\
\hline$\geqslant 23$ & $4.76 \%$ & $100 \%$ & $86.49 \%$ & & 0.95 \\
\hline$>23$ & $0 \%$ & $100 \%$ & $85.81 \%$ & & 1 \\
\hline
\end{tabular}


FIGURE 1 Receiver operating characteristic (ROC) curve of the tuberculin skin test to predict QuantiFERON-TB results.

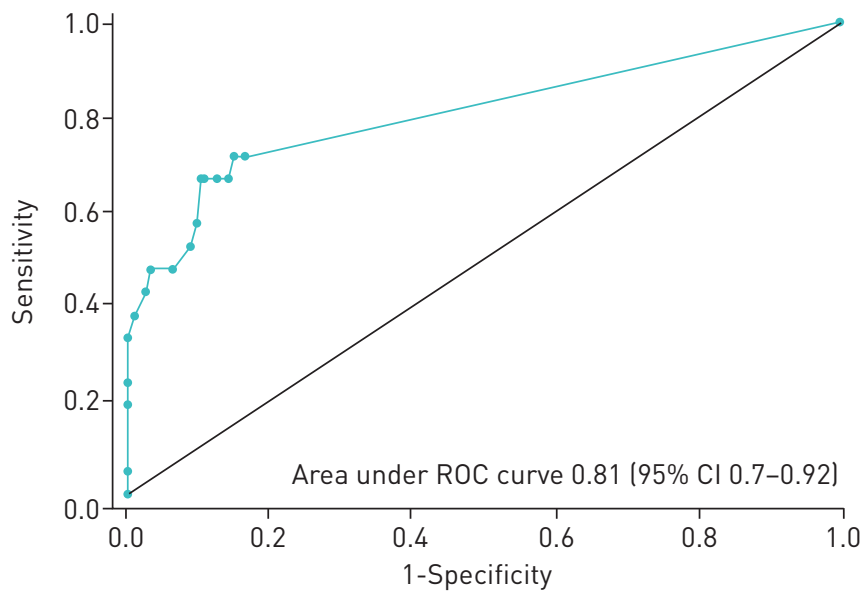

\section{Discussion}

Results from this sample of cancer patients showed a moderate agreement between tests used in the diagnosis of LTBI. Only a few studies worldwide have evaluated the agreement between these tests in cancer patients, mainly in patients with haematologic malignancies $[12,13,17,18]$. This research included subjects with solid cancers to provide broader information for these types of cancers.

Moon et al. [12] described, in patients with haematologic malignancies, that agreement between these tests was slight (TST $\geqslant 5 \mathrm{~mm}$ versus $\mathrm{QFT} \kappa=0.08,95 \% \mathrm{CI}-0.06-0.24$; TST $\geqslant 10 \mathrm{~mm}$ versus $\mathrm{QFT} \kappa=0.15,95 \%$ CI -0.004-0.31). RichelDI et al. [13] compared these tests in immunocompromised patients, some of them with haematologic malignancies, finding a moderate agreement (TST versus QFT $\kappa=0.65$; TST versus T-SPOT .TB $\kappa=0.4)$. A study conducted in patients undergoing chemotherapy found a fair agreement between QFT and TST ( $\kappa=0.25, \mathrm{p}=0.007)$; patients with TST $<10 \mathrm{~mm}$ underwent a booster by a second application of tuberculin and the booster test had stronger agreement with QFT $(\kappa=0.72, \mathrm{p}=0.001)$ suggesting that the booster is more comparable to QFT in cancer patients [14]. Although the precision of results was affected by the suspension of enrolment, the agreement between tests in the present study was moderate $(\kappa=0.43)$, which is consistent with other studies and highlights the limitations of these tests, since each one classifies different individuals as infected. Lacking a suitable concordance, we can conclude that these tests are not equivalent, but not which one is better for LTBI diagnosis.

The reasons for discordance between these tests are poorly understood. Both tests evaluate the host immunological memory against the mycobacteria, but it is likely that they measure different parameters of the immune response; it is suggested that IGRAs evaluate a recent exposure while TST evaluates a remote infection [19]. On the other hand, QFT results have been shown to be variable in repeated measurements, particularly when the results are around the manufacturer-recommended cut-off point $[9,20]$. Defects in manufacturing, sample processing delays, eradication of tuberculous infection and within-person biological variation in the production of interferon- $\gamma$ have been identified as sources of variability $[9,21]$. The lack of reproducibility of QFT affects its ability to define the diagnosis and its intra and intertest agreement.

FIGURE 2 Box-and-whisker plots showing the magnitude of antigen-nil response in QuantiFERON-TB results according to tuberculin skin test (TST) positivity.

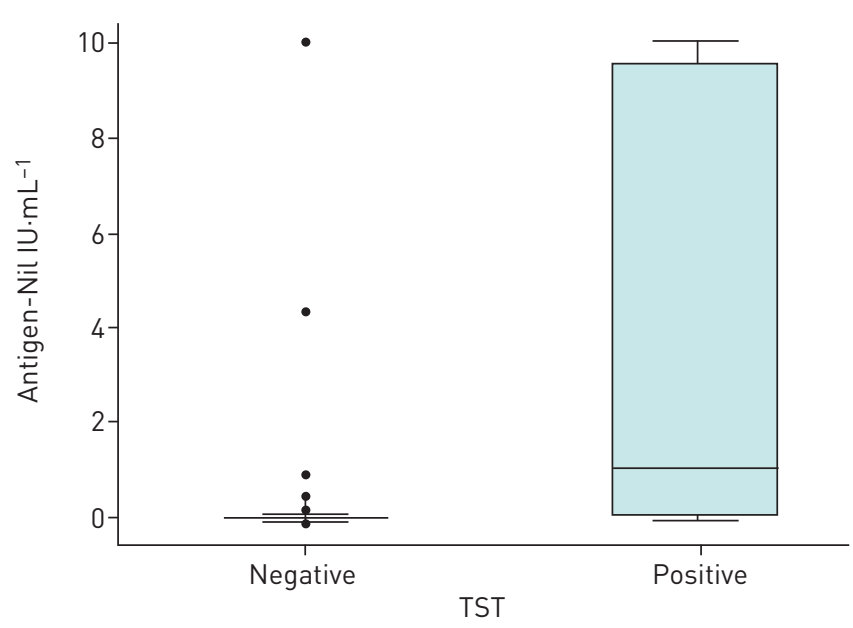


The National Health and Nutrition Examination Survey identified some factors related to the chance of having discordance between tests, such as age, sex, treatment for LTBI, and lymphocyte count [20]. We could not validate any predictor associated with discordance of the results.

One of the most relevant questions is whether the cut-off points of these tests to define positivity are suitable. It should be kept in mind that in the case of TST the cut-off points were set arbitrarily and in the case of QFT seems to favour their lack of reproducibility, it is feasible that their modification will change the agreement of tests $[9,22,23]$. In the present study, we explored different cut-off points to reach a better agreement between TST and QFT. In this population, a cut-off point $\geqslant 8 \mathrm{~mm}$ of the TST seems to better predict the QFT results, as well as a cut-off point $\geqslant 1 \mathrm{IU} \cdot \mathrm{mL}^{-1}$ of the antigen response in the QFT seems to better predict the TST results; however, the use of these cut-off points had only a marginal change in the agreement of tests. This exploratory analysis and available data suggest that cut-off points of test results should be reassessed [23, 24].

QFT Gold Plus is a new generation test that removes the TB7.7 antigen and includes an additional tube with antigens capable of stimulating interferon- $\gamma$ production by $\mathrm{CD} 8^{+} \mathrm{T}$-cells; recent data suggest that this test may have a slight improvement in sensitivity compared with QFT Gold In-Tube, but it does not seem to reduce test variability [25-28].

Colombia is a country of middle prevalence for tuberculosis infection, but there are no overall data on the frequency of LTBI. A study conducted in two men's prisons found a prevalence of TST positivity of 66\% [29]. We estimated a LTBI prevalence of $21.5 \%$ in cancer patients, defining LTBI as the positivity of any of the diagnostic tests considering they could identify different kinds of infected patients. The World Health Organization recommends either TST or IGRAs for LTBI diagnosis [30, 31]. Due to IGRAs higher cost and the fact that most of their studies have been carried out in high-income countries, their use is not recommended in middle or low-income countries such as Colombia [32].

One of the limitations of this study is that we did not test a booster with a second application of tuberculin, so we could not prove whether it correlated better with QFT [14]. Second, the weight of factors likely associated with discordance could not be ascertained with these results, possibly because of the low number of cases of LTBI. Third, cancer statuses may indicate varying degree of immunological statuses; 4 out of 10 patients included had a cancer status of partial or complete remission, therefore possibly a lower degree of immunosuppression. Fourth, this study was conducted in a developing country with a moderate prevalence of tuberculosis, where vaccination with BCG is almost universal (Colombia has $>80 \%$ coverage of BCG vaccination in newborns), so caution should be exercised about generalising these data to other settings; however, vaccination with BCG is expected not to cause false-positive test results for TST in Colombian adults because it is given just once at birth [31, 33, 34].

There is no gold standard for LTBI diagnosis. Diagnosis and treatment of people with LTBI could improve global tuberculosis care, but better diagnostic tools are needed. With new research on mycobacterial genomics and transcriptomics, the development of better diagnostic biomarkers is expected in the future [35]. For the moment, more studies are needed to evaluate the performance of the IGRAs and TST in populations at risk and the factors involved in their lack of agreement. Studies like this should be replicated in populations with a high prevalence of tuberculosis and with a low-income, in order to establish policies on targeted screening according to each population's needs.

\section{Conclusions}

Our findings suggest that the TST and QFT have a moderate agreement in cancer patients. Our study also provides information about the limitations on the interpretation of current cut-off points of test results. According to our data, the prevalence of LTBI in Colombia is substantial in cancer patients, and the potential implications of infection on the natural history of cancer should not be disregarded. Further research aimed at determining the performance of diagnostic strategies, such as sequential testing or the tuberculin booster is necessary.

\section{Conflict of interest: None declared.}

\section{References}

1 World Health Organization (WHO). Date last accessed: November 26, 2018. Date last updated: February 28, 2019. www.who.int/tb/publications/global_report/en.

2 Menzies NA, Wolf E, Connors D, et al. Progression from latent infection to active disease in dynamic tuberculosis transmission models: a systematic review of the validity of modelling assumptions. Lancet Infect Dis 2018; 18: e228-e238.

3 Sester M, van Leth F, Bruchfeld J, et al. Risk assessment of tuberculosis in immunocompromised patients. A TBNET study. Am J Respir Crit Care Med 2014; 190: 1168-1176.

4 Cheng MP, Abou Chakra CN, Yansouni CP, et al. Risk of active tuberculosis in patients with cancer: a systematic review and meta-analysis. Clin Infect Dis 2017; 64: 635-644.

5 Dobler CC, Cheung K, Nguyen J, et al. Risk of tuberculosis in patients with solid cancers and haematological malignancies: a systematic review and meta-analysis. Eur Respir J 2017; 50: 1700157. 
6 Mack U, Migliori GB, Sester M, et al. LTBI: latent tuberculosis infection or lasting immune responses to M. tuberculosis? A TBNET consensus statement. Eur Respir J 2009; 33: 956-973.

$7 \quad$ Huebner RE, Schein MF, Bass JB. The tuberculin skin test. Clin Infect Dis 1993; 17: 968-975.

8 American Thoracic Society. Targeted tuberculin testing and treatment of latent tuberculosis infection. Am J Respir Crit Care Med 2000; 161: S221-S247.

9 Pai M, Denkinger CM, Kik SV, et al. Gamma interferon release assays for detection of Mycobacterium tuberculosis infection. Clin Microbiol Rev 2014; 27: 3-20.

10 Diel R, Goletti D, Ferrara G, et al. Interferon- $\gamma$ release assays for the diagnosis of latent Mycobacterium tuberculosis infection: a systematic review and meta-analysis. Eur Respir J 2011; 37: 88-99.

11 Redelman-Sidi G, Sepkowitz KA. IFN- $\gamma$ release assays in the diagnosis of latent tuberculosis infection among immunocompromised adults. Am J Respir Crit Care Med 2013; 188: 422-431.

12 Moon SM, Lee SO, Choi SH, et al. Comparison of the QuantiFERON-TB Gold In-Tube test with the tuberculin skin test for detecting latent tuberculosis infection prior to hematopoietic stem cell transplantation. Transpl Infect Dis 2013; 15: 104-109.

13 Richeldi L, Losi M, D’Amico R, et al. Performance of tests for latent tuberculosis in different groups of immunocompromised patients. Chest 2009; 136: 198-204.

14 Mohammad HA, Esmail MA, Abdelftah MT, et al. Comparison of the two-step tuberculin skin test and QuantiFERON-TB Gold in-Tube test in the screening of latent tuberculosis infection in cancer patients. Egypt $J$ Chest Dis Tuberc 2015; 64: 681-688.

15 Cantor AB. Sample-size calculations for Cohen's kappa. Psychol Methods 1996; 1: 150-153.

16 Landis JR, Koch GG. The measurement of observer agreement for categorical data. Biometrics 1977; 33: 159-174.

17 Piana F, Codecasa LR, Cavallerio P, et al. Use of a T-cell-based test for detection of tuberculosis infection among immunocompromised patients. Eur Respir J 2006; 28: 31-34.

18 Stefan DC, Dippenaar A, Detjen AK, et al. Interferon-gamma release assays for the detection of Mycobacterium tuberculosis infection in children with cancer. Int J Tuberc Lung Dis 2010; 14: 689-694.

19 Pai M, Dheda K, Cunningham J, et al. T-cell assays for the diagnosis of latent tuberculosis infection: moving the research agenda forward. Lancet Infect Dis 2007; 7: 428-438.

20 Ghassemieh BJ, Attia EF, Koelle DM, et al. Latent tuberculosis infection test agreement in the National Health and Nutrition Examination Survey. Am J Respir Crit Care Med 2016; 194: 493-500.

21 Detjen AK, Loebenberg L, Grewal HMS, et al. Short-term reproducibility of a commercial interferon gamma release assay. Clin Vaccine Immunol 2009; 16: 1170-1175.

22 Tagmouti S, Slater M, Benedetti A, et al. Reproducibility of interferon gamma (IFN- $\gamma$ ) release assays. A systematic review. Ann Am Thorac Soc 2014; 11: 1267-1276.

23 Nemes E, Rozot V, Geldenhuys H, et al. Optimization and interpretation of serial QuantiFERON testing to measure acquisition of Mycobacterium tuberculosis infection. Am J Respir Crit Care Med 2017; 196: 638-648.

24 Winje BA, White R, Syre $\mathrm{H}$, et al. Stratification by interferon- $\gamma$ release assay level predicts risk of incident TB Thorax 2018; 73: 652-661.

25 Barcellini L, Borroni E, Brown J, et al. First independent evaluation of QuantiFERON-TB Plus performance. Eur Respir J 2016; 47: 1587-1590.

26 Barcellini L, Borroni E, Brown J, et al. First evaluation of QuantiFERON-TB Gold Plus performance in contact screening. Eur Respir J 2016; 48: 1411-1419.

27 Hoffmann H, Avsar K, Göres R, et al. Equal sensitivity of the new generation QuantiFERON-TB Gold plus in direct comparison with the previous test version QuantiFERON-TB Gold IT. Clin Microbiol Infect 2016; 22: 701-703.

28 Knierer J, Gallegos Morales EN, Schablon A, et al. QFT-Plus: a plus in variability? - Evaluation of new generation IGRA in serial testing of students with a migration background in Germany. J Occup Med Toxicol 2017; 12 : 1.

29 Rueda ZV, Arroyave L, Marin D, et al. High prevalence and risk factors associated with latent tuberculous infection in two Colombian prisons. Int J Tuberc Lung Dis 2014; 18: 1166-1171.

30 Rangaka MX, Wilkinson KA, Glynn JR, et al. Predictive value of interferon- $\gamma$ release assays for incident active tuberculosis: a systematic review and meta-analysis. Lancet Infect Dis 2012; 12: 45-55.

31 World Health Organization (WHO). Date last accessed: October 26, 2018. Date last updated: February 19, 2018 www.who.int/tb/publications/2018/latent-tuberculosis-infection/en.

32 World Health Organization (WHO). Date last accessed: June 14, 2018. Date last updated: June 16, 2012. www. who.int/tb/publications/tb-igras-statement/en.

33 Zwerling A, Behr MA, Verma A, et al. The BCG world atlas: a database of global BCG vaccination policies and practices. PLoS Med 2011; 8: e1001012.

34 Ministerio de Salud y Protección Social. SISPRO - Sistema Integrado de Información de la Protección Social. Date last accessed: July 9, 2019. www.sispro.gov.co/central-gestion-del-conocimiento/Pages/Construya-su-consultaVacunacion.aspx.

35 Singhania A, Wilkinson RJ, Rodrigue M, et al. The value of transcriptomics in advancing knowledge of the immune response and diagnosis in tuberculosis. Nat Immunol 2018; 19: 1159-1168. 